\title{
Effects of seasonal changes in the ionospheric conductances on magnetospheric field-aligned currents
}

\author{
A. J. Ridley ${ }^{1}$ \\ Received 12 October 2006; revised 20 December 2006; accepted 26 December 2006; published 1 March 2007.
}

[1] Through the seasons of the year, the ionospheric potential doesn't change significantly, while the solar driven conductance changes dramatically. This means that the magnetospherically driven field-aligned currents must be significantly reduced in the winter hemisphere and enhanced in the summer hemisphere. It is shown that, if this simplistic view of the conductance is used, the ratio between the maximum winter FAC and maximum summer hemisphere FAC is on the order of a factor of four. FAC data shows that the true factor is closer to two. When a seasonally dependent auroral precipitation is used to increase the auroral conductance in the winter hemisphere, the ratio between the maximum FAC in the summer and winter is reduced to 1.6. It is shown that the measured hemispheric power does indeed show a seasonal difference, with the winter hemisphere receiving more power by approximately $3.8 \mathrm{GW}$ (about 20\%). Citation: Ridley, A. J. (2007), Effects of seasonal changes in the ionospheric conductances on magnetospheric field-aligned currents, Geophys. Res. Lett., 34, L05101, doi:10.1029/2006GL028444.

\section{Introduction}

[2] The magnetosphere and the ionosphere are non-linearly coupled together, with the ionospheric potential being driven by the field-aligned currents from the magnetosphere and the ionospheric conductance. Because the magnetospheric field lines are frozen to the plasma motion, and that motion can be described by the ionospheric potential, when the potential is altered because of the conductance, the fieldline motion in the magnetosphere is also changed. This can alter the pressure distribution in the magnetosphere, thereby redistributing the field-aligned currents, which effects the ionospheric potential. Through this self-consistent feedback, the magnetospheric pressure and field-aligned currents adjust to the ionospheric conductance and potential patterns [Fedder and Lyon, 1987; Peymirat et al., 2002; Ridley et al., 2003, 2004].

[3] It is well known that the ionospheric conductance changes as a function of solar zenith angle [e.g., Moen and Brekke, 1993], and therefore the summer and winter polar regions can have dramatically different conductances. De La Beaujardiere et al. [1991] showed that the ionospheric potential also changes as a function of season, but only by 20-30\%. Ridley et al. [2004] showed that some simulations of the global magnetosphere have too strong of a difference between the winter and summer hemispheres. They showed

\footnotetext{
${ }^{1}$ Center for Space Environment Modeling, University of Michigan, Ann Arbor, Michigan, USA.
}

Copyright 2007 by the American Geophysical Union. 0094-8276/07/2006GL028444 that when the dipole tilt is very large, the ratio of the potentials can be a factor of $2-4$, rather than the $\sim 1.3$, as De La Beaujardiere et al. [1991] showed. Ridley et al. [2002] discussed that in order to correct for this discrepancy, a seasonal component of the auroral conductance could be added. Liou et al. [2001] showed observationally that the aurora has a seasonal dependence, with the nightside precipitation being stronger in the winter than in the summer. They further showed that there is an enhancement in the aurora on the dayside in the summer, which would serve to further strengthen the conductance. They argue that the dayside enhancement is due to a strengthening of the upwards FAC, which would lead to more precipitating electrons, consistent with relationships such as described by Knight [1972]. Other studies have also shown that there are seasonal differences that are caused by sunlight conditions in the aurora [Newell and Meng, 1988; Newell et al., 1996, 1998].

[4] This letter examines the statistical ionospheric potential and conductance patterns to derive field-aligned current patterns. These field-aligned current patterns are compared to statistical patterns, to determine whether the empirical (or statistical) models are self-consistent with each other. A seasonally dependent auroral precipitation pattern is then explored to justify the use of such a relationship in a global magnetosphere-ionosphere model. It is then shown that the hemispheric power demonstrates such a seasonal dependence.

\section{Results}

[5] Figure 1 shows the Northern spring (left) summer (mid-left), fall (mid-right), and winter (right) ionospheric potential (top) derived from the [Weimer, 1996] model for the interplanetary magnetic field $B_{z}=-5 \mathrm{nT}$, with the solar wind velocity equal to $400 \mathrm{~km} / \mathrm{s}$, the ionospheric Pedersen conductance (middle) for an $F_{10.7}$ of 217 , and the fieldaligned currents (bottom) that are derived from these two patterns (see Goodman [1995], Amm [1996], and Ridley et al. [2004] for a description of how this can be done). The Pedersen conductance includes a small particle precipitation component, driven by the Fuller-Rowell and Evans [1987] model with a hemispheric power of one Giga-Watt. The solar EUV driven conductance is from AMIE and is described by Ridley and Kihn [2004]. The ionospheric potentials are observed to change by only a very small amount $(\sim 15 \%)$, while the conductance changes by almost a factor of 10 at the pole. Because the field-aligned currents are derived from the potential and the conductances (in this example), the maximum (minimum) FAC changes by about a factor of 3-4 (5-6) between winter and summer. 

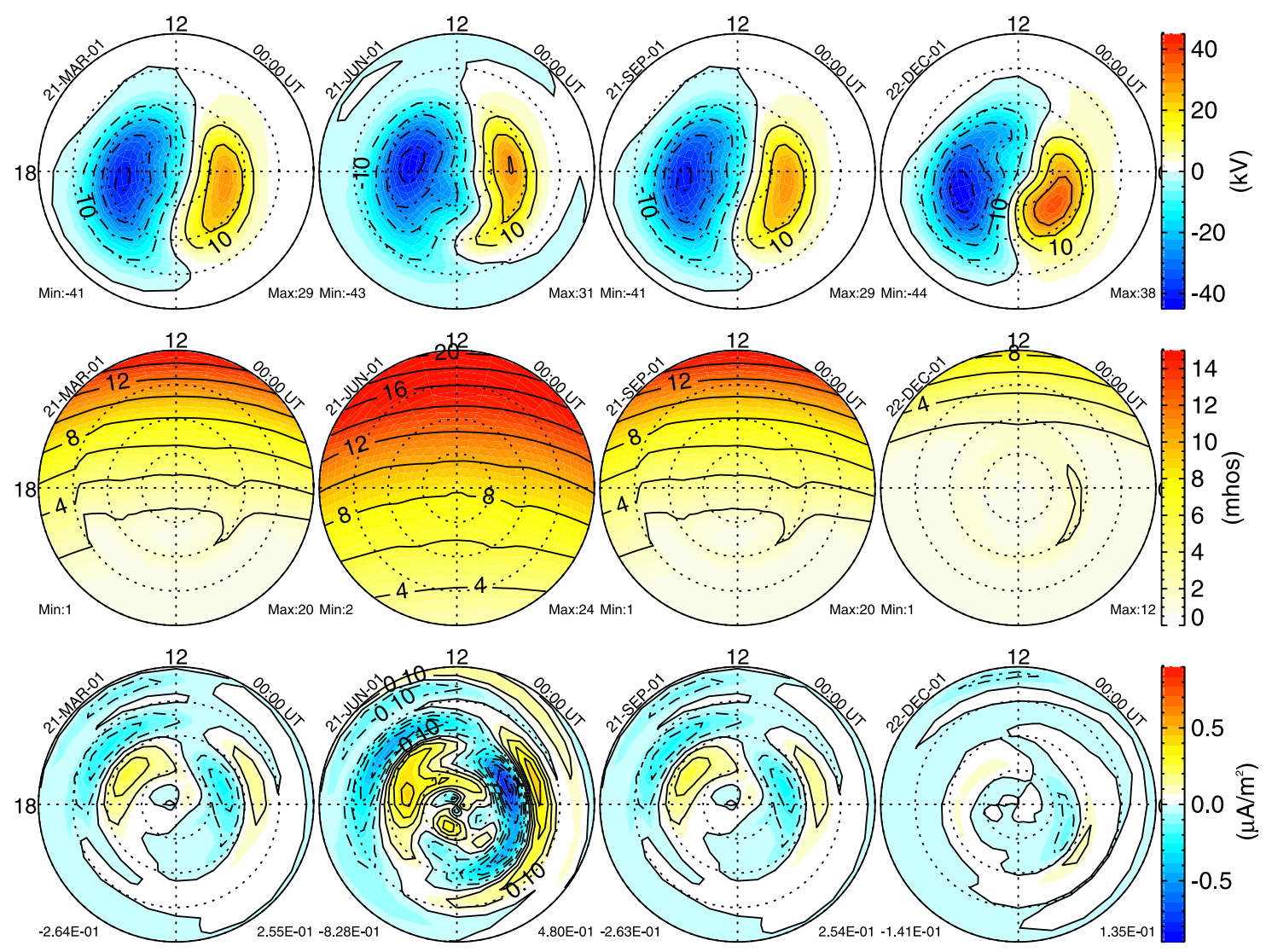

Figure 1. (top) The ionospheric potential, (middle) Pedersen conductance, and (bottom) derived field-aligned currents for northern spring, summer, fall, and winter (from left to right). The sun is towards the top in each figure with dawn at the right. The center is the northern magnetic pole with the outer circle being $50^{\circ}$ magnetic latitude. The minimum and maximum for each pattern is displayed also.

[6] Figure 2 further illustrates this tendency. The Pedersen conductance at the pole and in the post-midnight auroral zone, the cross polar cap potential, and the maximum FAC is shown as a function of time throughout a year, driven by the same conditions as described above. The maximum is shown, since it is related to downward precipitating electrons, and can be used to indicate the amount of precipitation that may be expected [Knight, 1972]. The hemispheric power is kept at a very low value throughout this time period (i.e. $1 \mathrm{GW}$ ). This accentuates the difference between winter and summer, since there is little auroral conductance to offset the lack of solar conductance in the winter.

[7] Using Figures 1 and 2, we can reverse the argument and address the question of how much FAC does the magnetosphere have to produce to generate the observed potential, given the conductance distribution. Figures 1 and 2 show that in order to get the potential to change by only $15 \%$ from summer to winter, given that the conductance at the pole changes by a factor of 10 , the magnetospheric driven field-aligned currents must change by a factor of $3-6$. The net amount of field-aligned current produced by the magnetosphere is most likely the same during the winter and summer solstices, but the ratio of the currents closing in the summer and winter hemispheres would have to differ significantly, with the majority of the current (about $75 \%$ $80 \%$ ) being driven through the summer hemisphere, and the winter hemisphere receiving a smaller amount of the current (about 20\%-25\%).

[8] The derived (or needed) field-aligned current maximum value can be compared to statistically derived fieldaligned current patterns. For example, Papitashvili et al. [2002] showed statistically derived field-aligned current maps from the Orsted satellite magnetic field measurements. They showed that, statistically, there is a factor of 1.6 between the summer maximum $\left(0.56 \mu \mathrm{A} / \mathrm{m}^{2}\right)$ and winter maximum $\left(0.35 \mu \mathrm{A} / \mathrm{m}^{2}\right)$ FACs. Wang et al. [2005] showed that the maximum FAC changes by approximately a factor of two from sunlit to darkness conditions on the dayside. From these studies, it can be concluded that the maximum FACs change from winter to summer by about a factor of two, while this study shows that it has to change by a factor of about four, in order for the potentials to change by only a small amount observed. This implies that the seasonal variation of the ionospheric conductance must not be correct.

[9] Therefore, this thought experiment on the FACs was repeated by altering the auroral conductance as a function of time throughout the year: using a small amount of auroral conductance during the summer and a large amount of auroral conductance during the winter. The hemispheric power used to drive the Fuller-Rowell and Evans [1987] patterns in the new experiment is shown in Figure 3. The 


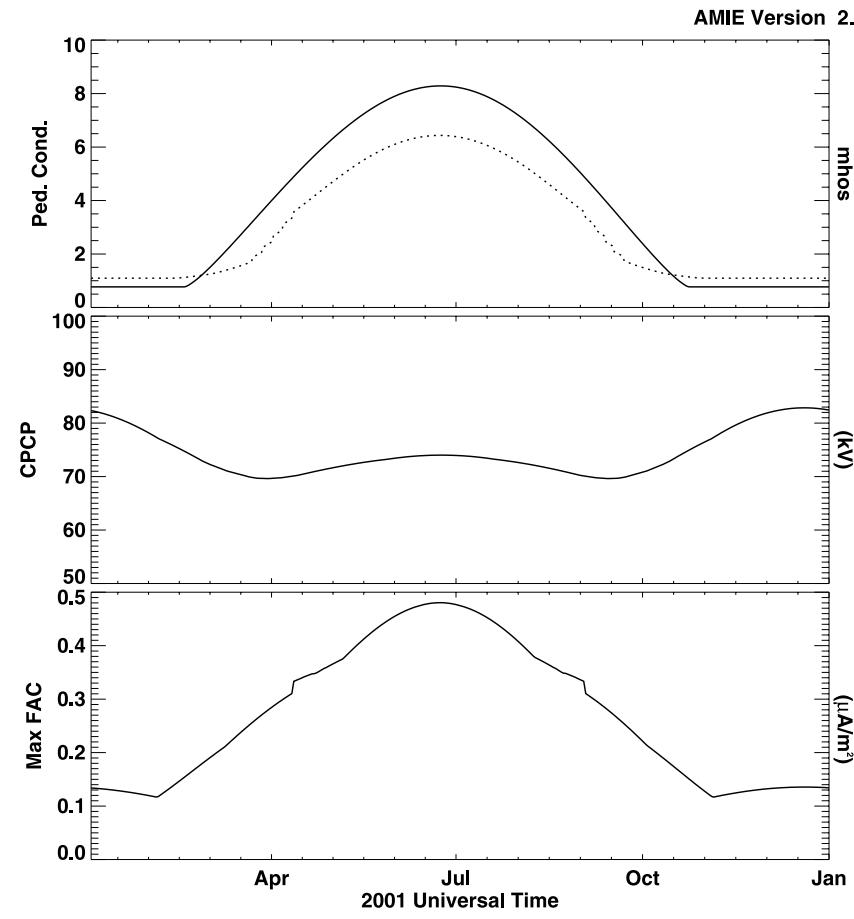

Figure 2. From top to bottom: the Pedersen conductance at the pole (solid) and the average Pedersen conductance between $60^{\circ}-70^{\circ}$ magnetic latitude and 00-06 magnetic local time (dotted); the cross polar cap potential; and the maximum of the derived field-aligned currents.

horizontal dotted lines show the activity levels in which the modeled patterns are changed (i.e. the hemispheric power index levels 1-4, from bottom to top, so, when the hemispheric power is between two of the dotted lines, the model doesn't change). For most of the winter and equinox seasons, the hemispheric power level stays the same, while it drops during the summer season.

[10] The new conductance and FAC patterns are shown in Figure 4. The potentials stay the same as in Figure 1, since nothing in the IMF or solar wind were altered. For the winter and equinox patterns there is a stronger auroral oval than in the summer (compared to Figure 1). The conductance in the midnight region is still strongest in the summer. The time variation of the aurora zone conductance and effect on the FAC is more clearly seen in Figure 5. The conductance at the pole is not changed over the unaltered run, while the auroral zone conductance is enhanced during the winter and equinox times. This enhanced conductance leaded to larger field-aligned currents, bringing the ratio between the maximum summer and winter FAC to 1.6, which is much more in line with the observed ratio. The summer field-aligned current patterns do not change for the runs (since the hemispheric power is the same, and hence the conductance is the same), while the Equinox and winter patterns do change. The increase of the winter FACs is what brings the ratio to be more in-line with other studies. The minimum FAC ratio between the seasons is still significantly larger then observed, though. While the first run (i.e. Figure 1) shows a ratio of 5.9 between the minimum FACs, the second runs (i.e. Figure 4) shows a ratio of 3.1 , which is much closer to the ratio of 1.8 that is observed by Papitashvili et al. [2002].

[11] The shape of the winter FAC patterns is consistent with other studies, also. For example, Wang et al. [2005] shows that the peak downward FAC in sunlit conditions is in 09-10 MLT sector, while Figure 4 shows the downward peak to be around 07 MLT in the summer. In the winter, the downward FAC peak is much broader and is between midnight and dawn, which is the same as in the darkness condition FACs reported by Wang et al. [2005].

\section{Discussion and Conclusions}

[12] In the previous section, it was shown that in order to have consistent changes in the field-aligned currents and potentials between the summer and winter seasons, there needs to be a seasonal dependence upon the auroral conductance to compensate for the change in the solar driven conductance. Ridley et al. [2002] described a need for this in an MHD model, while Liou et al. [2001] showed that precipitation on the nightside is enhanced during the winter, as would be expected from the results above. To further determine whether this finding is valid, the hemispheric power is examined.

[13] Figure 6 shows the hemispheric power measured by the NOAA satellites from 1979-2006 for the northern and southern hemispheres. The plots were derived by calculating a weekly median value for each day of the year (i.e. the median value within a seven day window centered on each day of the year), then calculating the mean daily value for all of the years. The median value was used for the first calculation to remove outlying points, although the properties of the plot do not change if the mean is used. It appears that there is a trend for lower hemispheric power in the summer and in the middle of winter in both hemispheres, with peaks near the equinoxes [e.g., Russell and McPherron, 1973]. There is also an approximate 27 day periodicity to the hemispheric power, which is more evident in the first half of the year. This is evidently the solar rotation. A more subtle effect is that the southern hemisphere tends to have less hemispheric power than the northern hemisphere in the first and last part of the year, while the northern hemisphere

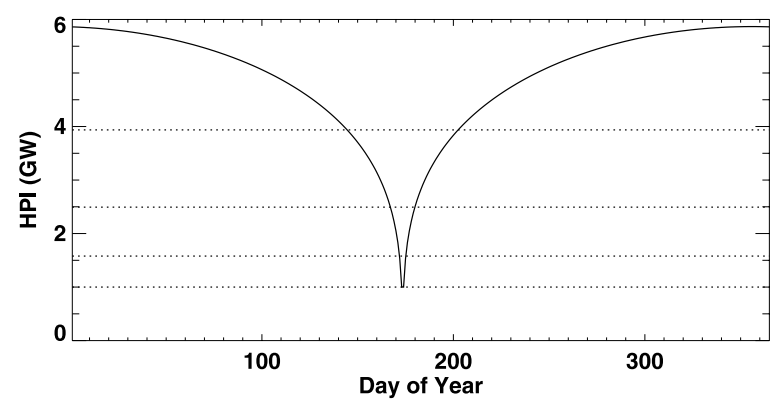

Figure 3. The northern hemispheric power used to drive the Fuller-Rowell and Evans [1987] model of the Pedersen conductance for the run shown in Figures 4 and 5. The dotted lines show the activity levels in which different models are used. 

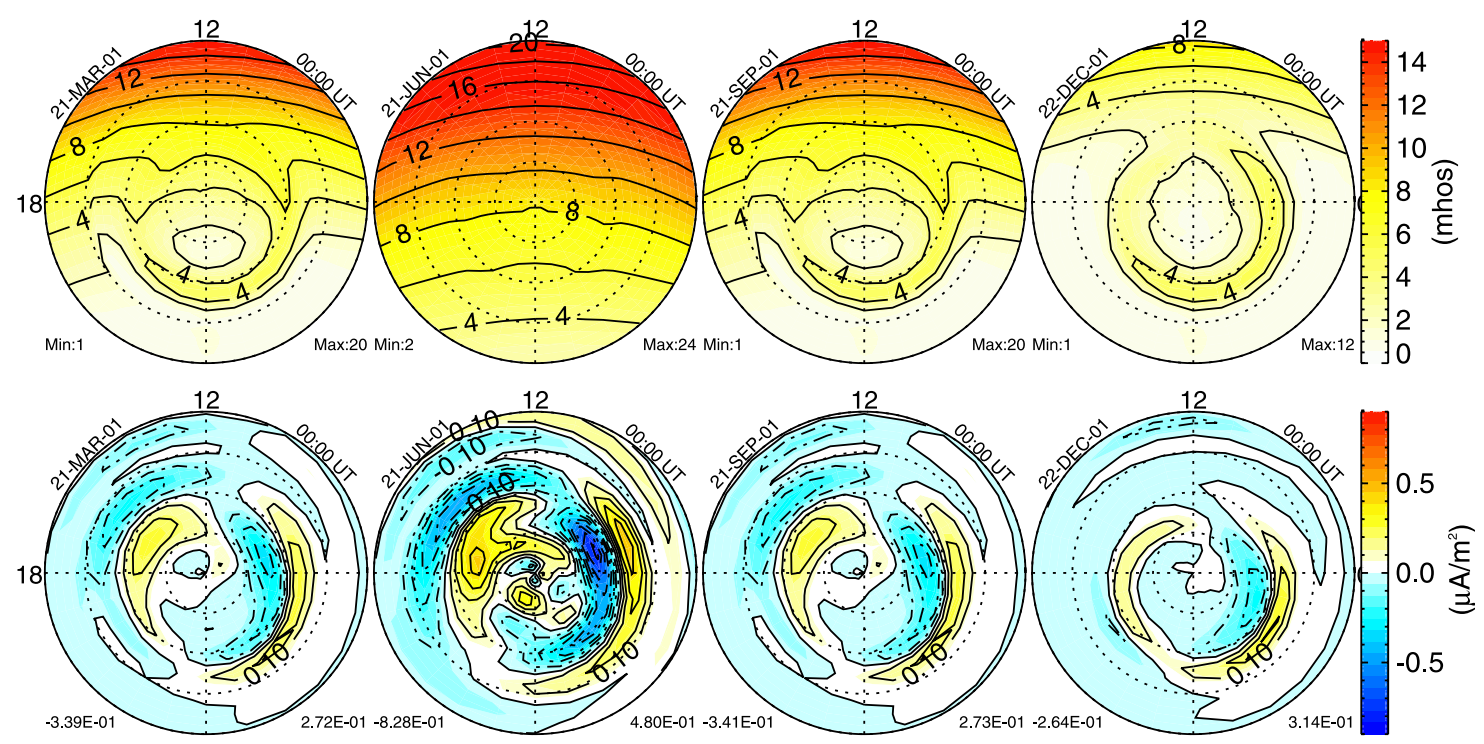

Figure 4. (top) The Pedersen conductance and (bottom) FAC in the same format as Figure 1 for the case when the HPI is changed over the seasons.

has less power in the middle of the year. This is shown more clearly in the bottom plot, which shows the difference between the northern and southern hemispheric powers. In addition, there is a cos fit to this line, with an amplitude of $1.82 \mathrm{GW}$, a mean of $0.82 \mathrm{GW}$, and a start day of December 22. This shows that statistically, there is about $3.6 \mathrm{GW}$ (or $20 \%$ ) more energy flowing into the winter hemisphere than the summer hemisphere (centered on the solstice), which is

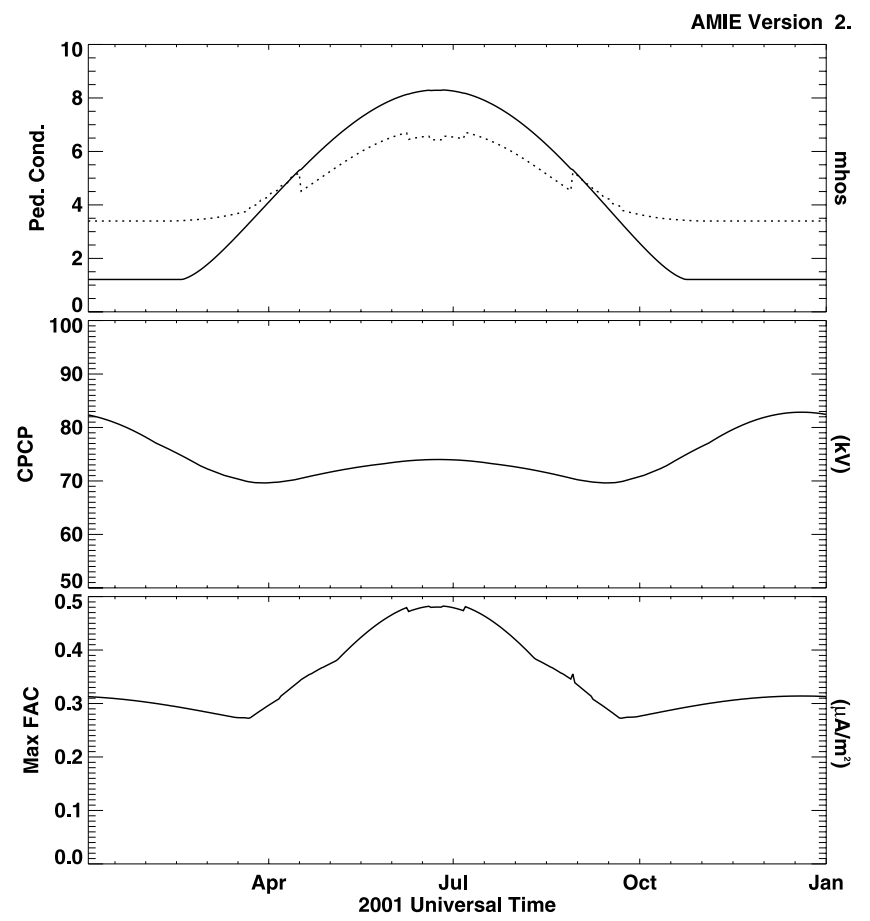

Figure 5. The same quantities as in Figure 2 for the case in which the HPI is altered over the seasons. consistent with the findings described in the previous section.

[14] In many global MHD codes, the Knight [1972] relation or other empirical formula are used to determine the strength of the discrete aurora based on the strength of the field-aligned currents [e.g., Raeder et al., 2001; Ridley et al., 2004]. This is problematic, since the summer hemisphere typically has more FAC than the winter hemisphere, leading to more precipitation in the summer than the winter (in the models), which is consistent with the findings by Liou et al. [2001] on the dayside, but is opposite to what is needed on the nightside, as described above.

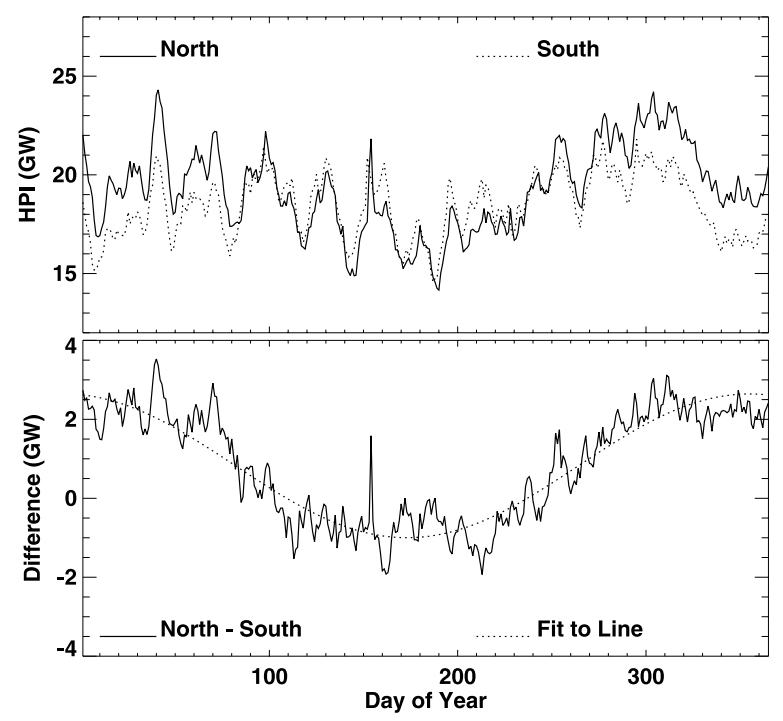

Figure 6. (top) Average northern and southern hemispheric power measured by the NOAA satellites from 1979-2006, (bottom) with the difference between them. 
[15] Acknowledgments. This research was supposed by NSF through grants ATM-0077555, ATM-0417839, and ATM-0325332 and NASA grant NNG04GK18G.

\section{References}

Amm, O. (1996), Comment on "A three-dimensional, iterative mapping procedure for the implementation of an ionosphere-magnetosphere anisotropic Ohm's law boundary condition in global magnetohydrodynamic simulations," Ann. Geophys., 14, 773.

De La Beaujardiere, O., D. Alcayde, J. Fontanari, and C. Leger (1991), Seasonal dependence of high-latitude electric fields, J. Geophys. Res., 96, 5723.

Fedder, J. A., and J. G. Lyon (1987), The solar wind-magnetosphereionosphere current-voltage relationship, Geophys. Res. Lett., 14, 880.

Fuller-Rowell, T. J., and D. S. Evans (1987), Height-integrated Pedersen and Hall conductivity patterns inferred from TIROS-NOAA satellite data, J. Geophys. Res., 92, 7606.

Goodman, M. L. (1995), A three-dimensional, iterative mapping procedure for the implementation of an ionosphere-magnetosphere anisotropic Ohm's law boundary condition in global magnetohydrodynamic simulations, Ann. Geophys., 13, 843.

Knight, S. (1972), Parallel electric fields, Planet. Space Sci., 21, 741.

Liou, K., P. T. Newell, and C.-I. Meng (2001), Seasonal effects on auroral particle acceleration and precipitation, J. Geophys. Res., 106, 5531.

Moen, J., and A. Brekke (1993), The solar flux influence of quiet-time conductances in the auroral ionosphere, Geophys. Res. Lett., 20, 971.

Newell, P. T., and C.-I. Meng (1988), Hemispherical asymmetry in cusp precipitation near solstice, J. Geophys. Res., 93, 2643.

Newell, P. T., C.-I. Meng, and K. M. Lyons (1996), Suppression of discrete aurorae by sunlight, Nature, 381, 766.

Newell, P. T., C.-I. Meng, and S. Wing (1998), Auroral frequency if independent of solar cycle in darkness and anti-correlated in sunlight, Nature, 342, 393.

Papitashvili, V. O., E. Friis-Christiansen, and T. Neubert (2002), A new model of field-aligned currents derived from high-precision satellite magnetic field data, Geophys. Res. Lett., 29(14), 1683, doi:10.1029/ 2001 GL014207.
Peymirat, C., A. D. Richmond, and R. G. Roble (2002), Neutral wind influence on the electrodynamic coupling between the ionosphere and the magnetosphere, J. Geophys. Res., 107(A1), 1006, doi:10.1029/ 2001JA900106.

Raeder, J., R. L. McPherron, L. A. Frank, S. Kokubun, G. Lu, T. Mukai, W. R. Paterson, J. B. Sigwarth, H. J. Singer, and J. A. Slavin (2001), Global simulation of the Geospace Environment Modeling substorm challenge event, J. Geophys. Res., 106, 281.

Ridley, A. J., and E. A. Kihn (2004), Polar cap index comparisons with AMIE cross polar cap potential, electric field, and polar cap area, Geophys. Res. Lett., 31, L07801, doi:10.1029/2003GL019113.

Ridley, A. J., K. C. Hansen, G. Tóth, D. L. De Zeeuw, T. I. Gombosi, and K. G. Powell (2002), University of Michigan MHD results of the Geospace Global Circulation Model metrics challenge, J. Geophys. Res., 107(A10), 1290, doi:10.1029/2001JA000253.

Ridley, A. J., T. I. Gombosi, D. L. De Zeeuw, C. R. Clauer, and A. D. Richmond (2003), Ionospheric control of the magnetospheric configuration: Neutral winds, J. Geophys. Res., 108(A8), 1328, doi:10.1029/ 2002JA009464.

Ridley, A. J., T. I. Gombosi, and D. L. De Zeeuw (2004), Ionospheric control of the magnetospheric configuration: Conductance, Ann. Geophys., 22, 567-584.

Russell, C. T., and R. L. McPherron (1973), The magnetotail and substorms, Space Sci. Rev., 15, 205.

Wang, H., H. Lühr, and S. Y. Ma (2005), Solar zenith angle and merging electric field control of the field-aligned currents: A statistical study of the Southern Hemisphere, J. Geophys. Res., 110, A03306, doi:10.1029/ 2004JA010530.

Weimer, D. R. (1996), A flexible, IMF dependent model of high-latitude electric potential having "space weather" applications, Geophys. Res. Lett., 23, 2549.

A. J. Ridley, Center for Space Environment Modeling, University of Michigan, 1416 Space Research Building, 2455 Hayward Street, Ann Arbor, MI 48109-2143, USA. (ridley@umich.edu) 